\title{
Space-Time Block Codes from Co-ordinate Interleaved Orthogonal Designs
}

\author{
Md. Zafar Ali Khan and B. Sundar Rajan \\ Dept. of Electrical Comm. Engg., \\ Indian Institute of Science, Bangalore, India. \\ \{zafar@protocol., bsrajan@\}ece.iisc.ernet.in
}

\begin{abstract}
In this paper we present space-time block codes called 'Co-ordinate interleaved orthogonal designs' (CIOD). These codes have low decoding complexity; same as that of the codes from complex orthogonal designs (COD). Rate 1 CIOD exist for two, three and four transmit antennas and rate $3 / 4$ CIOD exist for five, six, seven and eight transmit antennas while COD exists only for two antennas and generalized COD (GCOD) of rate $3 / 4$ are known to exist for three and four transmit antennas only. The maximum mutual information of these codes is also calculated.
\end{abstract}

\section{CIOD}

A generalized complex linear processing orthogonal design [1](GCLPOD): of size $N$ of rate $k / p$, in variables $x_{i}, i=$ $1, \cdots, k$ is a $p \times N$ matrix $\Theta\left(x_{1}, \cdots, x_{k}\right)$, such that (i) the entries of $\Theta\left(x_{1}, \cdots, x_{k}\right)$ are complex linear combinations of $x_{i}, i=1, \cdots, k$ and their conjugates and (ii) $\Theta^{\dagger}\left(x_{1}, \cdots, x_{k}\right) \Theta\left(x_{1}, \cdots, x_{k}\right)=\mathcal{D}$, where $\mathcal{D}$ is a diagonal matrix whose entries are a linear combination of $\left|x_{i}\right|^{2}, i=1, \cdots, k$ with all strictly positive real coefficients and $\Theta^{\dagger}$ is the complex transpose conjugate of $\Theta$. When $k=p=N$ we obtain complex linear processing orthogonal design (CLPOD) [1].

Definition 1.1 A co-ordinate interleaved orthogonal design (CIOD)- of size $N$ of rate $k / p$, in variables $x_{i}, i=1, \cdots, k$ is a $p \times N$ matrix $S\left(x_{1}, \cdots, x_{k}\right)$, such that

$$
S\left(x_{1}, \cdots, x_{k}\right)=\left[\begin{array}{cc}
\Theta\left(\tilde{x}_{1}, \cdots, \tilde{x}_{k / 2}\right) & 0 \\
0 & \Theta\left(\tilde{x}_{k / 2+1}, \cdots, \tilde{x}_{k}\right)
\end{array}\right]
$$

where $\Theta\left(x_{1}, \cdots, x_{k / 2}\right)$ is a GCLPOD of size $N / 2$ of rate $k / p$ $\tilde{x}_{i}=\operatorname{Re}\left\{x_{i}\right\}+\mathbf{j} \operatorname{Im}\left\{x_{(i+k / 2)_{k}}\right\}$ and where $(a)_{k}$ denotes $a$ $(\bmod k)$

Examples of rate 1, CIOD's for $N=2,4$ are

$$
\begin{gathered}
S\left(x_{1}, x_{2}\right)=\left[\begin{array}{cc}
\tilde{x}_{1} & 0 \\
0 & \tilde{x}_{2}
\end{array}\right], \\
S\left(x_{1}, \cdots, x_{4}\right)=\left[\begin{array}{cccc}
\tilde{x}_{1} & \tilde{x}_{2} & 0 & 0 \\
-\tilde{x}_{2}^{*} & \tilde{x}_{1}^{*} & 0 & 0 \\
0 & 0 & \tilde{x}_{3} & \tilde{x}_{4} \\
0 & 0 & -\tilde{x}_{4}^{*} & \tilde{x}_{3}^{*}
\end{array}\right] .
\end{gathered}
$$

Theorem 1.1 A rate 1 , co-ordinate interleaved orthogonal design of size $N$ exists if and only if $N=2,3$ or 4

A rate 1 CIOD for $N=3$ can be obtained form $N=4$, CIOD by deleting one of the columns. If $\Theta$ is a GCLPOD of size 4, rate $3 / 4$ then we have rate $3 / 4$ CIOD codes for $\mathrm{N}=5,6,7,8$.

${ }^{1}$ This work was partly supported by IISc-DRDO programme on Advanced Research in Mathematical Engineering through a research grant to B. S. Rajan.

\section{ENCODING \& DECODING USING CIOD}

The transmission scheme for these designs for a system involving $N$ transmit and $M$ receive antennas is as follows: let $k b$ bits arrive at the encoder in a given time slot. The encoder selects $k$ symbols, $s_{i}, i=1, \cdots, k$ from a complex constellation $\mathcal{A},|\mathcal{A}|=2^{b}$. Then setting $x_{i}=s_{i}, i=1, \cdots, k$, the encoder populates the transmission matrix for the corresponding number of transmit antennas. The received signal in matrix notation is given by,

$$
V=S H+\mathcal{N}
$$

where $V \in \mathbb{C}^{p \times M}$ is the received signal matrix, $S \in \mathbb{C}^{p \times N}$ is the transmission matrix and $\mathcal{N} \in \mathbb{C}^{p \times M}$ is the additive noise matrix whose entries are i.i.d. complex Gaussian with zero mean and unit variance. $H \in \mathbb{C}^{N \times M}$ defines the channel matrix, such that the element in the $i$ th row and $j$ th column is $h_{i j}$, the channel response from transmitter $i$ to receiver $j$ and is complex Gaussian with zero mean and unit variance. The transmit power constraint is given by $\operatorname{tr}\left(S^{\dagger} S\right)=p$. Observe that CIOD consists of two CLPOD or GCLPOD. Therefore the decoder performs linear processing similar to that of CLPOD or GCLPOD (of size $N / 2$ ) over two block lengths of size $p / 2$ and then interleaves to decouple the transmitted symbols.

Observe that due to interleaving at the transmitter, there is an expansion of signal set involved and the transmitted signals from each transmit antenna are not from $\mathcal{A}$.

Theorem 2.2 CIOD's achieve full diversity order provided, no two signal points in the signaling constellation $\mathcal{A}$ have same co-ordinates, i.e., if $\left(x_{I}, x_{Q}\right)$ and $\left(y_{I}, y_{Q}\right)$ are signal points then $x_{I} \neq y_{I}$ and $x_{Q} \neq y_{Q}$.

This result links the performance of CIOD to Co-ordinate Product Distance (CPD) in accordance with the results on co-ordinate interleaved schemes like bit and co-ordinate interleaved coded modulation [2] and the references therein.

Though CIOD's have low decoding complexity, they do not achieve capacity even for rate 1 and $M=1$. If we define $C(N, M, \rho), C_{D}(N, M, \rho)$ and $C_{O}(N, M, \rho)$ as the capacity, maximum mutual information of CIOD and CLPOD respectively, for $N$ transmit and $M$ receive antennas at $\operatorname{SNR} \rho$ then

$$
\begin{aligned}
& C_{D}(2,1, \rho)=C(1,1, \rho)<C(2,1, \rho)=C_{O}(2,1, \rho), \\
& C_{O}(4,1, \rho)<C_{D}(4,1, \rho)=C(2,1, \rho)<C(4,1, \rho) .
\end{aligned}
$$

\section{REFERENCES}

[1] V.Tarokh, H. Jafarkhani and A.R. Calderbank, "Space-time block codes from Orthogonal designs," IEEE Trans. on Inform. Theory, Vol. 45, pp. 1456-1467, July 1999.

[2] Md. Zafar A. K. and B. Sundar Rajan, "Bit and co-ordinate interleaved coded modulation," Proc. of GLOBECOM'2000, pp. 1595-1599. 